\title{
Photoelectron spectrometer for attosecond spectroscopy of liquids and gases
}

\author{
I. Jordan, ${ }^{1}$ M. Huppert, ${ }^{1}$ M. A. Brown, ${ }^{2}$ J. A. van Bokhoven, ${ }^{3,4}$ and H. J. Wörner ${ }^{1, a)}$ \\ ${ }^{1}$ Laboratory of Physical Chemistry, ETH Zurich, Vladimir-Prelog-Weg 2, 8093 Zurich, Switzerland \\ ${ }^{2}$ Laboratory for Surface Science and Technology, Department of Materials, ETH Zurich, \\ Vladimir-Prelog-Weg 5, 8093 Zurich, Switzerland \\ ${ }^{3}$ Institute for Chemical and Bioengineering, ETH Zurich, Vladimir-Prelog-Weg 1, 8093 Zurich, Switzerland \\ ${ }^{4}$ Laboratory for Catalysis and Sustainable Chemistry, Paul Scherrer Institute, 5232 Villigen, Switzerland
}

(Received 11 September 2015; accepted 6 December 2015; published online 28 December 2015)

\begin{abstract}
A new apparatus for attosecond time-resolved photoelectron spectroscopy of liquids and gases is described. It combines a liquid microjet source with a magnetic-bottle photoelectron spectrometer and an actively stabilized attosecond beamline. The photoelectron spectrometer permits venting and pumping of the interaction chamber without affecting the low pressure in the flight tube. This pressure separation has been realized through a sliding skimmer plate, which effectively seals the flight tube in its closed position and functions as a differential pumping stage in its open position. A high-harmonic photon spectrometer, attached to the photoelectron spectrometer, exit port is used to acquire photon spectra for calibration purposes. Attosecond pulse trains have been used to record photoelectron spectra of noble gases, water in the gas and liquid states as well as solvated species. RABBIT scans demonstrate the attosecond resolution of this setup. (C) 2015 AIP Publishing LLC. [http://dx.doi.org/10.1063/1.4938175]
\end{abstract}

\section{INTRODUCTION}

Attosecond science has greatly contributed to our understanding of ultrafast electronic and nuclear dynamics in atoms and small molecules. ${ }^{1-8}$ Extending attosecond science to the liquid phase is expected to not only offer new insights into the influence of a surrounding solvent on electronic dynamics in molecules but also to allow studying ultrafast processes in chemical systems under ambient conditions. Examples include electronic dynamics specific to liquid phase, e.g., electron transfer, solvation dynamics including solvated electrons, and solvent reorganization. Through the process of high-harmonic generation, intense short-wavelength radiation in the form of attosecond pulse trains or even isolated attosecond pulses is available from table-top laser sources. ${ }^{9,10}$

For a long time, photoelectron spectroscopy was limited to low-vapor-pressure samples due to the low mean free path of electrons in high-pressure environments on the one hand and the low-pressure requirements of the photoelectron detectors on the other hand. The absorption of extremeultraviolet (XUV) radiation by most gases additionally limits the pressure in the interaction region to $10^{-3}$ mbar at most. Liquids typically have vapor pressures around 1-30 mbar at room temperature, making themselves an unlikely target to be studied under high-vacuum conditions. This so-called pressure gap has finally been bridged in the late 1990s by the invention of the liquid-microjet technique. ${ }^{11,12}$ In combination with liquid-nitrogen cold traps and a differential-pumping stage, this liquid filament can flow steadily in high vacuum while maintaining pressures low enough for photoelectron detection.

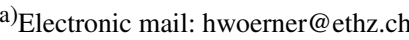

The time-resolved liquid-jet photoelectron spectroscopy experiment is depicted schematically in Fig. 1. Generally, any kind of solution can be studied with such a setup, ${ }^{11}$ although high-vapor-pressure liquids restrict the usable jet diameters and skimmer openings. During the last years, photoelectron spectra of water and water-soluble compounds have been studied extensively; ${ }^{13-18}$ however, toluene, ${ }^{19}$ methanol, and ethanol ${ }^{20}$ have proven to be suitable as well. Since the solvent is a minor restriction, any kind of solute from salts over transition metal complexes to nanoparticles can be studied, the photoelectron signal being mainly determined by the solubility and surface-affinity of the target compound and the sensitivity being often limited by the overlap with the solvent photoelectron spectrum. Recently, several groups have presented experimental setups

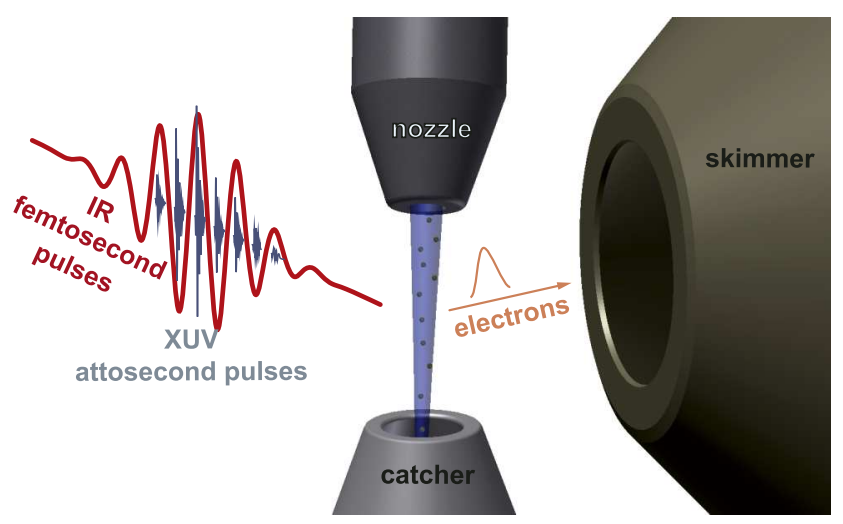

FIG. 1. General design of the liquid-phase attosecond-time-resolved photoelectron spectroscopy experiment. A quartz nozzle injects a liquid filament into vacuum. Phase-locked XUV and IR pulses generate an electronic wave packet in the continuum which enters the time-of-flight spectrometer through the skimmer opening. 
combining liquid microjets with a photoelectron spectrometer, mostly using synchrotron radiation ${ }^{21-23}$ or sum-frequency generation from laser radiation $^{5,24,25}$ to ionize water and solvated molecules. Also, first photoelectron spectra have been reported using a high-harmonic radiation source. ${ }^{26,27}$ However, time-dependent measurements were so far restricted to the dynamics of solvated electrons, ${ }^{5,28}$ excited state relaxation of DNA bases, ${ }^{14,29}$ and charge-transfer-to-solvent processes. ${ }^{30}$

In this article, we present the combination of attosecond metrology with the liquid-microjet technique. We describe a magnetic-bottle-time-of-flight spectrometer for ultrafast timeresolved photoelectron spectroscopy of gases and liquids coupled to an attosecond beamline containing a highharmonic radiation source. This experimental setup enables us to perform pump-probe experiments with attosecond pulse trains (RABBIT ${ }^{31}$ ) accessing attosecond temporal information.

\section{INSTRUMENT DESIGN}

The photoelectron spectrometer was designed to measure both gas-phase and liquid-phase time-dependent photoelectron spectra. A magnetic-bottle ${ }^{32}$ setup has been chosen over a field-free version for the following reasons. Short acquisition times on the scale of less than $5 \mathrm{~s}$ per spectrum are desired in order to perform attosecond time-resolved measurements with scan ranges of about $10 \mathrm{fs}$ in less than $20 \mathrm{~min}$. However, space-charge effects have to be avoided which sets an upper limit to the number of electrons produced by each laser shot in the interaction region. Additionally, depending on the choice of metal filters or XUV mirrors, the photon flux can be very low. A magnetic bottle typically collects half of the photoelectron emission sphere (approximately $2 \pi \mathrm{sr}$ ), whereas in a field-free design with a $910 \mathrm{~mm}$-long flight-tube and a detector diameter of $27 \mathrm{~mm}$, the collection solid angle would be limited to $0.7 \mathrm{msr}$. Thus, a magnetic bottle mitigates the signal limitations imposed by space-charge effects and/or flux limitations through a high collection efficiency resulting in short acquisition times. The resulting layout of the liquid-jet setup is shown in Figure 2(a).

\section{A. Magnetic bottle}

Magnetic-bottle parts are colored in orange in Figure 2(a) and comprise the SmCo permanent magnet with a magnetic field strength of $1 \mathrm{~T}$, holding a conical iron tip, and the four-layer solenoid with a $0.3 \mathrm{~mm}$ wire diameter, which generates a homogeneous magnetic field of $1 \mathrm{mT}$ along the flight tube. The magnetic bottle is thus divided into two parts, a high-pressure interaction region with a divergent magnetic field hosting the jet and a low-pressure flight tube where the magnetic field is homogeneous. The differential pumping stage between both pressure regions is realized through a gold-coated aluminum skimmer with an aperture of $1 \mathrm{~mm}$, mounted on a sliding plate. The permanent magnet is mounted on an xyz-manipulator and can be positioned very precisely using micrometer screws, such that the cylindrical axes of the permanent magnet and the solenoid overlap. The microchannel plate (MCP) detector with a single-event response of $0.9 \mathrm{~ns}$ marks the end of the electron flight path and is placed inside the solenoid such that the magnetic field lines are parallel all the way to the detector. A mesh (30 lines/in., copper wires, $90 \%$ transmission) has been installed in front of the detector in order to accelerate electrons to kinetic energies above $300 \mathrm{eV}$, where the MCP is more sensitive compared to $\sim 10 \mathrm{eV}$. The distance between the interaction region and the microchannel plate detector is around $910 \mathrm{~mm}$, and can be changed by shifting the spectrometer with respect to the focus position by $\pm 5 \mathrm{~mm}$.

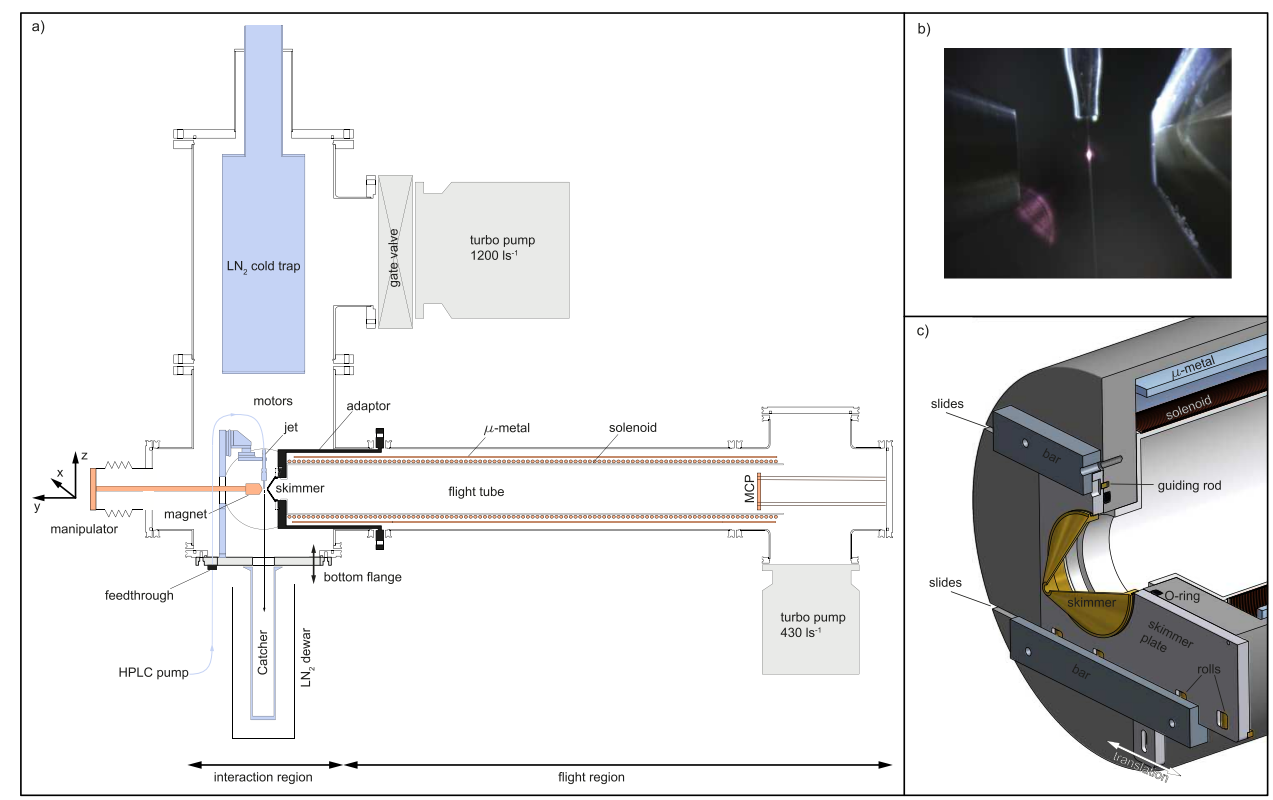

FIG. 2. (a) Cross section of the liquid-jet photoelectron spectrometer. (b) Liquid jet flowing at $2 \cdot 10^{-4}$ mbar, the skimmer is on the right and the magnet tip on the left. The bright spot is IR light being scattered by the non-laminar part of the jet. (c) Schematic representation of the skimmer closing mechanism. 


\section{B. Liquid microjet}

Spectrometer parts associated with the liquid jet, such as the jet holder, the positioning motors, the liquid-nitrogen cold trap, the catcher and the jet itself, are highlighted in blue in Figure 2(a). Using a HPLC pump, the solution to be studied is pumped from a reservoir through 1/16 in. PEEK tubing and is injected into the interaction chamber through a nozzle. Quartz nozzles for liquid jet applications in vacuum are commercially available in various sizes and jet diameters between 15 and $50 \mu \mathrm{m}$ are typically used in our setup. The nozzle can be positioned with $1 \mu \mathrm{m}$ precision piezo-motors, which are mounted to a holder on the bottom flange of the interaction chamber. ${ }^{21}$ The liquid filament streams into a stainless steel catcher which is cooled on the atmospheric side with liquid nitrogen and freezes the solution as it hits the catcher walls. In order to reach pressures below $1 \cdot 10^{-3} \mathrm{mbar}$, liquid nitrogen cold traps are inevitable in the interaction chamber. The liquid-jet nozzle can easily be replaced by a gas nozzle, such that switching between gas-phase and liquid-phase experiments is possible within a few hours.

\section{Operation of the liquid jet under vacuum}

The liquid microjet has to be started under atmospheric pressure and it is therefore necessary to pump and vent the interaction region each time the experiment starts or ends, respectively. Consequently, the skimmer design was determined by two considerations. First, the skimmer has to function as a differential-pumping stage during the measurement and second, a closing mechanism is desirable that preserves the vacuum in the flight tube during the venting and pumping processes of the interaction chamber. The design of such a closing mechanism is shown in Figure 2(c). It is based on an aluminum plate with an opening on the lefthand side, on top of which the skimmer is mounted and is flat on the right-hand side. Using a mechanical, one-axis feedthrough, this skimmer plate can be translated along the laser beam axis, thereby placing either the skimmer or the flat part of the aluminum plate in front of the flight-tube opening. Two bars press the aluminum plate against an O-ring which is located in a groove on the adaptor. In order to ensure the reproducibility of the skimmer position, a guiding mechanism has been installed. The aluminum plate has two guiding rods on the back side, which are guided along slides in the adaptor. It is very important to maintain a minimum mechanical pressure on the sliding aluminum plate in order to squeeze the O-ring and to maintain a high vacuum in the flight tube during pumping and venting and also during translation of the aluminum plate. At the same time, the friction during translation has to be minimized. These challenges have been addressed by mounting rolls on the upper and lower part of the aluminum front plate, which make the only contact to the bars such that there is a negligible friction between the aluminum plate and the bars. Therefore, the only friction is resulting from the shear strain between the aluminum plate and the O-ring and any kind of twist occurring between the guides and the sliding rods. The latter has been minimized by using long rods made from brass.
In the first step of pumping the interaction chamber, an independent scroll pump is used to reach prevacuum pressures of about 0.1 mbar. Afterwards, the gate valve to the turbo pump, which is running continuously can be opened while the valve to the scroll pump has to be closed simultaneously. Both turbo pumps are connected via the same prevacuum system and the pressure in the flight tube is only affected marginally by the pumping of the interaction chamber.

\section{Attosecond beamline}

In order to perform time-resolved experiments, the liquidjet spectrometer has to be connected to a pump-probe setup. $^{33,34}$ The actively stabilized beamline part is shown in Fig. 3 and is described in more detail elsewhere. ${ }^{35}$ Briefly, the output of an amplified Ti:Sa femtosecond laser centered at $800 \mathrm{~nm}$ is focused by an off-axis parabolic mirror into a gas cell consisting of three differential pumping stages. High-order harmonics are being created in the pinhole of the first pumping stage. Due to the much lower divergence of the XUV radiation, it can be separated from the residual IR radiation by a perforated recollimating off-axis parabolic mirror after entering the first chamber of the beam-line. The XUV beam propagates undisturbed into the second beam-line chamber where it is being reflected under grazing incidence on diamond-like-carbon coated mirrors, thereby also passing a gold-coated toroidal mirror which images the XUV-generation area into the interaction chamber. The separated residual IR is redirected into the third chamber by two mirrors on piezostages, which control the delay between the XUV pulse train and the IR pulse. The IR beam reflected from a perforated focusing mirror in the third chamber arrives collinearly with the XUV in the interaction chamber of the photoelectron spectrometer. Spatial and temporal overlaps of both foci can be adjusted on a CMOS camera by inserting a pickoff-mirror into the recombined beam path. An XUV-photon spectrometer attached to the exit port of the photoelectron spectrometer allows recording of high-harmonic photon spectra simultaneously with photoelectron spectra. Isolating the XUV from the IR beam enables a separate optical manipulation of both beams, including filtering harmonic spectra with metal foils in the XUV path and chopping the IR beam for single-shot referencing. Motorized irises have been placed in both beam paths in order to independently adjust the intensities.

Using the setup depicted in Fig. 3, we measured RABBIT traces in various noble gases, and gaseous and liquid water. Recording the high-harmonic spectrum simultaneously allows to retrieve the actual harmonic photon energies and intensities used for ionization and to perform the calibration of the time-of-flight axis of the photoelectron spectra.

\section{SPECTROMETER CHARACTERIZATION}

Xenon is very well suited for spectrometer calibration purposes using a high-harmonic radiation source. Photoelectron signals originate mostly from the $5 \mathrm{p}$ shell which possesses two well separated spin-orbit components. The ionization 


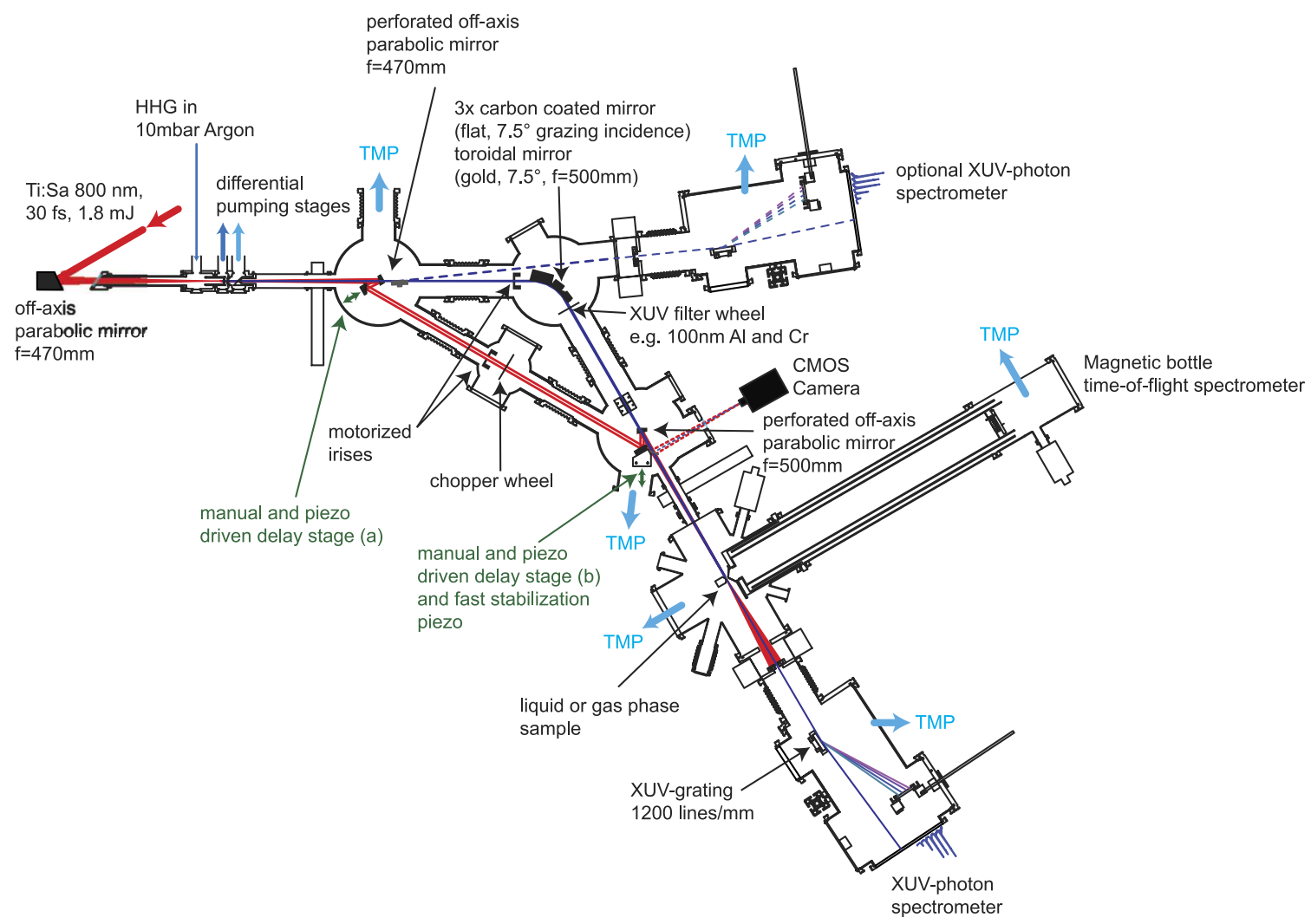

FIG. 3. Complete experimental setup including a gas cell for high-harmonic generation, an actively stabilized beam-line, a photoelectron spectrometer equipped with a liquid jet and a XUV spectrometer. Turbomolecular pumps are marked with TMP.

cross section of the $5 \mathrm{~s}$ orbital is significantly smaller for the energy ranges covered in the present experiment (typically up to $40 \mathrm{eV}$ ), such that no signals associated with the $5 \mathrm{~s}$ electrons appear in the photoelectron spectra. A typical timeof-flight spectrum is presented in Fig. 4 covering harmonic orders 11-25. For data analysis and spectrometer-response characterization, the time-of-flight axis has to be converted to an energy axis.

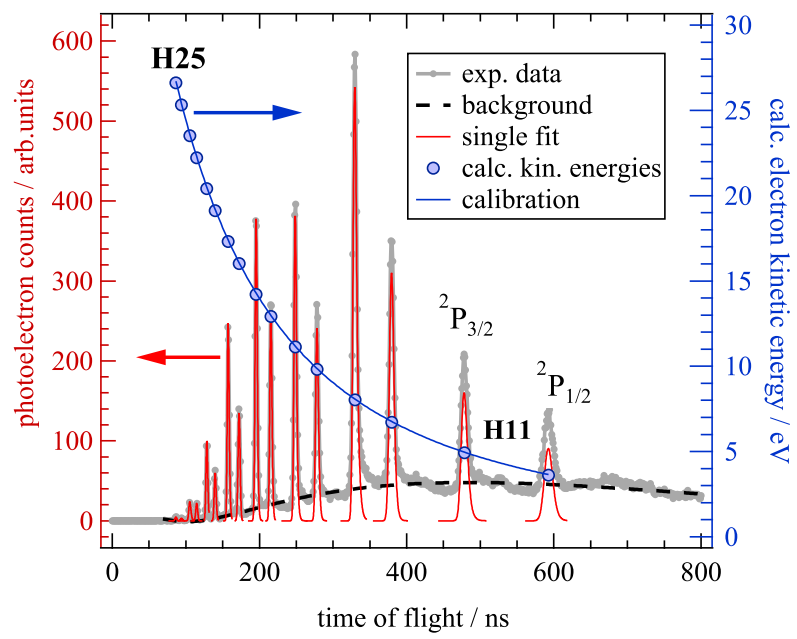

FIG. 4. Time-of-flight spectrum of xenon (gray markers) and single peak fit (red lines) using Equation (1). The electron's kinetic energy has been calculated for both spin-orbit components using a central wavelength of $795 \mathrm{~nm}$ and plotted against the measured time of flight (blue circles). The fitting curve (blue line) of the calibration procedure agrees well with the calculated kinetic energies (filled blue circles).

\section{A. Calibration and conversion}

The magnetic-bottle spectrometer is calibrated using the photoelectron spectrum generated by ionizing xenon with high-order harmonics. The convolution of a Gaussian with the product of an exponential and the Heaviside function, $\Theta(x)$,

$$
\begin{aligned}
f(x)= & \frac{A}{2 \pi \sigma} \exp \left(-\frac{1}{2} \frac{\left(x-x_{0}\right)^{2}}{\sigma^{2}}\right) * \\
& {\left[\exp \left(-\frac{x-x_{0}}{\tau}\right) \cdot \Theta\left(x-x_{0}\right)\right], }
\end{aligned}
$$

is fitted to each photoelectron peak in the time domain. Using this fit function, we can account for the exponential tail occurring in time-of-flight signals. The background signal most likely originates from secondary electrons created through electron-impact ionization by high-energy primary photoelectrons. The extracted photoelectron flight times refer to the maximum of each fitted peak. Kinetic energies, $E_{\text {kin }}$, calculated from harmonic energies and binding energies are plotted against the measured time-of-flight values, $t$, as retrieved from the above-mentioned fit. The conversion function

$$
E_{\text {kin }}=\frac{m}{2}\left(\frac{s}{t-t_{0}}\right)^{2}+E_{0}
$$

is then fitted to the calculated kinetic energies, with the drift length $s$, the temporal offset $t_{0}$, and the energy offset $E_{0}$ being fit parameters. A temporal offset has to be fitted since the laser trigger is used for starting the acquisition. An aluminum filter with a thickness of $100 \mathrm{~nm}$ does not transmit harmonic orders 
below the 11th order of $800 \mathrm{~nm}$ and is used for unambiguous assignment of harmonic orders to the photoelectron signals. Based on Equation (2), the flight-time axis is then converted into a kinetic energy axis using the calibration parameters. The Jacobian has to be taken into account when converting the photoelectron signal from the time-of-flight to the energy axis in order to preserve the signal integrals.

Harmonic energies used in the calibration procedure have been calculated using the center of mass wavelength of the infrared spectrum $(795 \mathrm{~nm})$ and xenon binding energies of $12.130 \mathrm{eV}$ for the ${ }^{2} \mathrm{P}_{3 / 2}$ component and $13.436 \mathrm{eV}$ for the ${ }^{2} \mathrm{P}_{1 / 2}$ component. ${ }^{36}$ The resulting calibration parameters and their standard deviation from the fitting procedure for the example shown here are $s=(0.914 \pm 0.001) \mathrm{m}$, $t_{0}=(-210.4 \pm 0.4) \mathrm{ns}$, and $E_{0}=(0.06 \pm 0.01) \mathrm{eV}$. Figure 4 indicates that the chosen calibration function fits well to the calculated kinetic energies and measured flight times. The calibration parameters depend on the alignment of the liquid/gas jet, laser beam, and magnetic bottle and have to be determined after each alignment procedure on a daily basis.

\section{B. Energy resolution}

Due to the parallelization mechanism of electron trajectories in the magnetic bottle, the photoelectron peaks exhibit an exponential tail towards decreasing kinetic energies. Using the same kind of exponentially modified Gaussian function as described in the calibration part, the peak position on the energy axis, $E_{0}$, the full-width-at-half-maximum (FWHM) values of the asymmetric line shape, $\Delta E$ can be determined and are shown in Fig. 5. For some lines, the fit gives very small values for the exponential tail (H25 and $\mathrm{H} 19$ ${ }^{2} \mathrm{P}_{1 / 2}$ ), which reflects essentially Gaussian peak profiles.

The widths of the photoelectron bands result from the convolution of the following contributions: the electronic response of the detection, the high-harmonic spectral width, the instrument broadening function of the magnetic bottle spectrometer, and a possible natural line width. The amplified

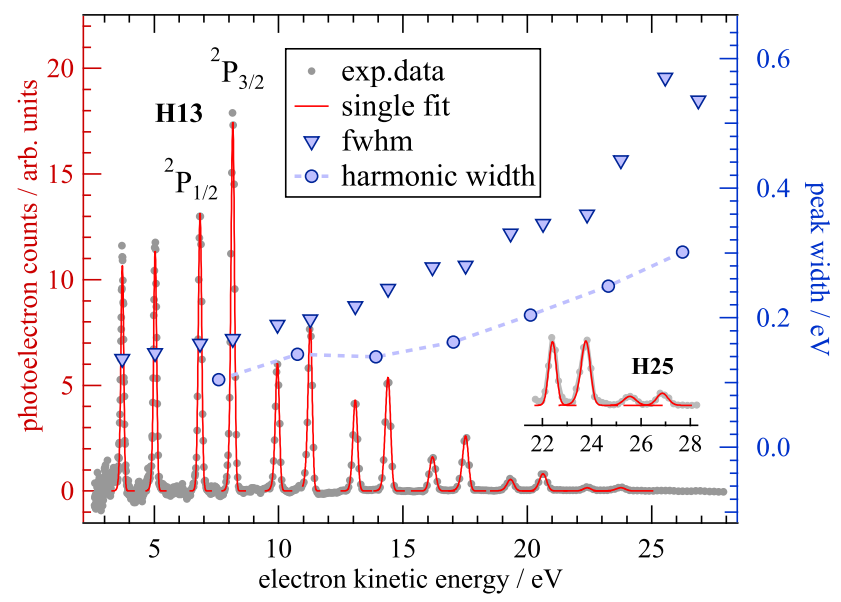

FIG. 5. Converted, background-subtracted photoelectron spectrum of xenon, ionized with harmonic orders 11-25. Blue triangles represent the FWHM of each photoelectron peak, and the blue circles correspond to the Gaussian widths of the harmonic radiation determined from the high-harmonic signal on the MCP image in Fig. 6.

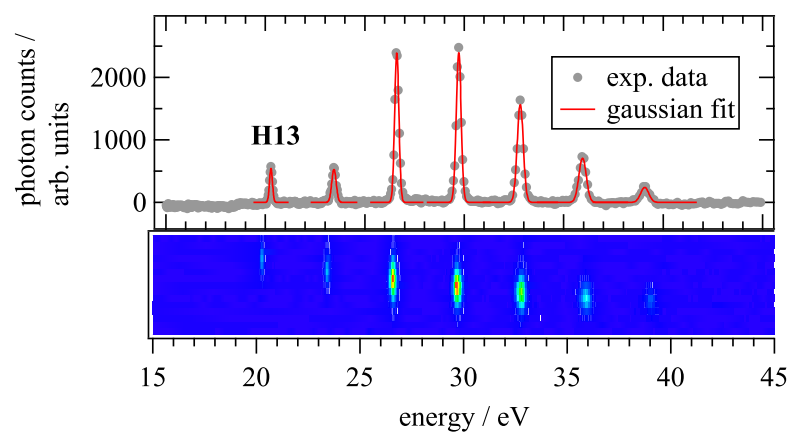

FIG. 6. High-harmonic spectrum recorded using the photon spectrometer, showing harmonic orders 13-25. The lower panel shows the MCP image; the upper panel results from integrating the lower panel along the vertical axis.

single-electron response of the MCP in the time domain can be fitted with a Gaussian function and has a full width at halfmaximum of $0.9 \mathrm{~ns}$. This response cannot be neglected given the fact that the temporal width of the time-of-flight signals is between 3 and $15 \mathrm{~ns}$ for harmonic orders 11-25. However, the maximum digitizer sampling rate is 1 gigasample/s, which restricts the minimum peak width to $1 \mathrm{~ns}$ and therefore the maximum resolution to $0.15 \mathrm{eV} / 26.87 \mathrm{eV}=0.006$ (25th harmonic) or $0.009 \mathrm{eV} / 3.72 \mathrm{eV}=0.002$ (11th harmonic), respectively.

In order to determine the second contribution, i.e., the high-harmonic spectral widths, an XUV-photon spectrometer was attached to the exit port of the photoelectron spectrometer (see Fig. 3). Harmonics enter the photon spectrometer through a $0.5 \mathrm{~mm}$ slit and are dispersed by a grating with 1200 lines/mm onto a MCP with a phosphor screen. Figure 6 shows the high-harmonic spectrum for the same harmonics which have been used to produce the photoelectron spectrum shown in Figs. 4 and 5. Harmonic order 11 has not been recorded because it was diffracted onto a position which was not covered by the sensitive area of the MCP. Harmonic orders have been assigned by translating the MCP along the image plane towards lower photon energies and using the aluminum filter to identify harmonic order 11. Using the measured central wavelength of $795 \mathrm{~nm}$ and neglecting ionization-induced spectral shifts, the camera pixels can be converted to an energy axis.

The energy resolution $\Delta E / E$ of the photoelectron spectrometer for the given range of photon energies without the correction for the harmonic widths, $\Delta E_{h}$, and detection broadening is summarized in Table I. As is clearly seen from the values, the $\Delta E / E$ resolution is a constant value of 0.02 for this particular energy range; however, it is increasing below $5 \mathrm{eV}$.

\section{INSTRUMENT PERFORMANCE}

\section{A. RABBIT scans}

Using a continuous gas nozzle with a $30^{\circ}$ tapered hole with a diameter of $10 \mu \mathrm{m}$, RABBIT scans of argon have been recorded (Fig. 7). The RABBIT experiment has been described in detail elsewhere. ${ }^{31}$ Briefly, the XUV pulse train, 
TABLE I. Photoelectron peak information as a function of harmonic order. Given are the resolution, absolute $(\Delta E)$ and relative $(\Delta E / E)$ to the kinetic energy $E$, and the spectral widths of the harmonics as extracted from the photon spectrometer camera image $\left(\Delta E_{h}\right)$.

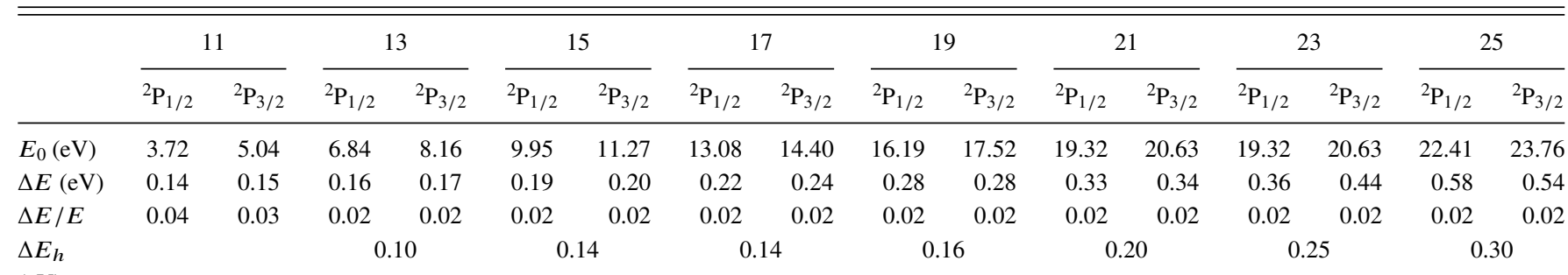

$(\mathrm{eV})$

generated in the gas cell, is separated from and delayed against the remaining IR. XUV and IR beams are then recombined and focussed into the interaction chamber. Each high-order harmonic in the XUV train generates a photoelectron signal, the photoelectron bands therefore being separated by twice the fundamental (here $800 \mathrm{~nm}$ ) photon energy, $3.1 \mathrm{eV}$. The presence of the weak assisting IR field gives rise to sidebands (SBs) located between the principal peaks. Due to quantum-path interference, the sidebands oscillate at twice the fundamental laser frequency, i.e., $1.35 \mathrm{fs}$, as the delay between XUV and IR pulses is scanned. This oscillation is clearly seen in Fig. 7. Due to the very small spin-orbit splitting (0.18 $\mathrm{eV}$ ) in argon, the two components are not resolved in these spectra. Extracting the phases of each sideband oscillation and the intensity of the photoelectron bands allows to reconstruct the electric field $E(t)$ of the attosecond pulse train according to

$$
E(t)=\sum_{i} A_{i} \exp \left(-\mathrm{i}\left(\omega_{\mathrm{i}} \mathrm{t}-\Delta \phi_{\text {xuv }}\right)\right)
$$

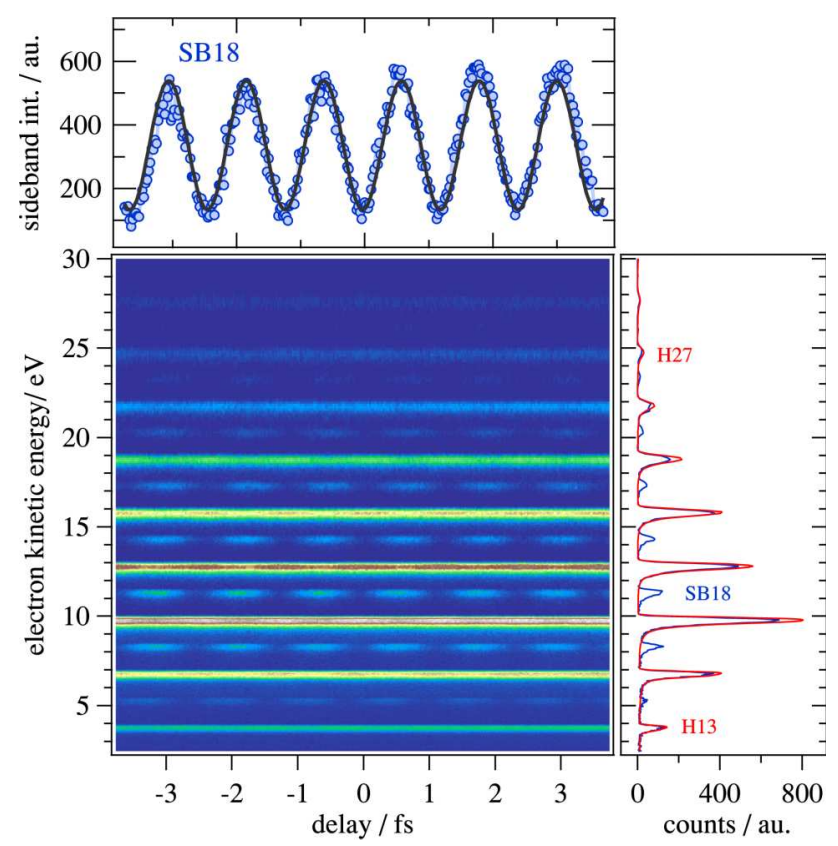

FIG. 7. RABBIT trace of argon. On the right-hand side the photoelectron spectrum with (blue line) and without (red line) IR is shown. The top graph shows a cut through the RABBIT trace for sideband order 18, marked with a yellow dashed line. where $A_{i}$ are the electric field amplitudes, $\omega_{i}$ the frequencies of the harmonics and $\phi_{\text {xuv }}$ their phases. The latter are related to the harmonic emission times $\tau$ by

$$
\tau=\frac{\Delta \phi_{\mathrm{xuv}}}{2 \omega_{0}}
$$

where $\omega_{0}$ represents the frequency of the infrared radiation. Equation (3) describes the superposition of monochromatic XUV pulses with infinite lengths, an assumption made to describe the quantum path interference by means of secondorder time-dependent perturbation theory. Integrating the sideband intensity along the energy axis and fitting a sine curve to the sideband oscillation provides the relative phases of the oscillations. Those can be decomposed into three contributions, the XUV spectral phases for each harmonic, the Wigner delay, and the measurement-induced phase, the latter two often being referred to as the atomic phase, ${ }^{37,38}$

$$
\tau=\tau_{\mathrm{xuv}}+\tau_{\mathrm{Wigner}}+\tau_{\mathrm{cc}} .
$$

The atomic phase can be calculated for systems like argon and used to determine the XUV phases. ${ }^{39}$ The XUV phases and emission times extracted from the scan shown in Fig. 7 are shown in Fig. 8. A quadratic dependence of the phase on the photon energy reflects the chirp of the reconstructed attosecond pulse, which is also shown in Fig. 8. Better synchronization of harmonic emission times results in less chirped pulses and therefore shorter pulse durations of the attosecond bursts.

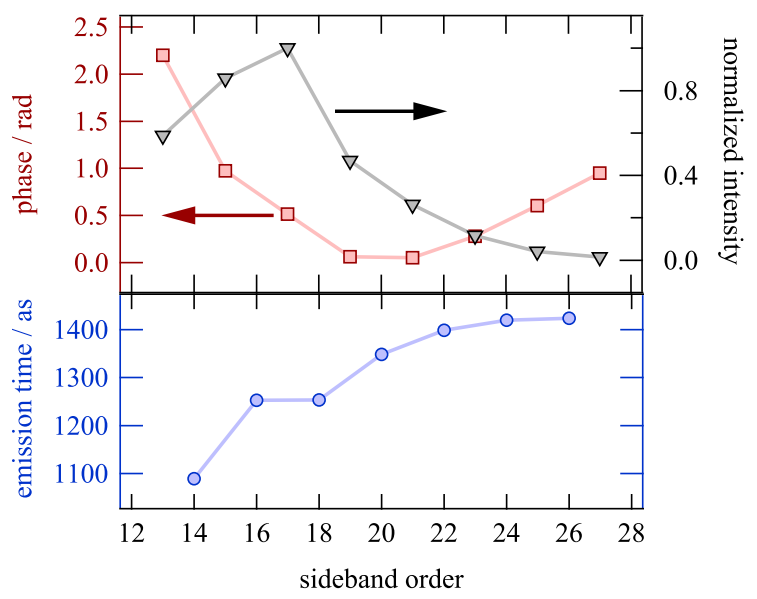

FIG. 8. High-harmonic emission times and phases. The quadratic phase dependence on the harmonic order reflects the chirp of the XUV pulse train. 


\section{B. Photoelectron spectra of gas-phase water}

A liquid-water microjet serves as a convenient source for measuring water in the gas and liquid phases under identical conditions. Moving the jet in the direction perpendicular to the XUV propagation axis allows for the acquisition of only gas-phase or both gas- and liquid-phase signals. The water gas pressure decreases according to $r_{0} / r$ for $r>r_{0}$, where $r_{0}$ is the jet radius and $r$ is the distance from the center of the jet. ${ }^{12,42}$ The maximum gas pressure is found directly at the vacuum-water interface where it is equal to the water vapour pressure $\left(23.4 \mathrm{mbar}\right.$ at $\left.20^{\circ} \mathrm{C}\right)$. Alternatively, a leak valve can be used to fill the interaction chamber up to a gas pressure of $5 \cdot 10^{-3}$ mbar. The differential pumping stage always maintains the pressure below $1 \cdot 10^{-5} \mathrm{mbar}$ at the MCP detector. This background filling is particularly useful when high electron count rates are expected from the ionization cross section and from the high-harmonic flux.

The photoelectron spectrum presented in Fig. 9 was generated in the background-filled setup with harmonic orders 11-15, which were isolated from a high-harmonic spectrum generated in argon using a $100 \mathrm{~nm}$ tin filter. Based on flux measurements performed on a different beamline equipped with the same semi-infinite gas cell, we estimate the photon flux to be $10^{5}$ photons/shot/harmonic. The valence-

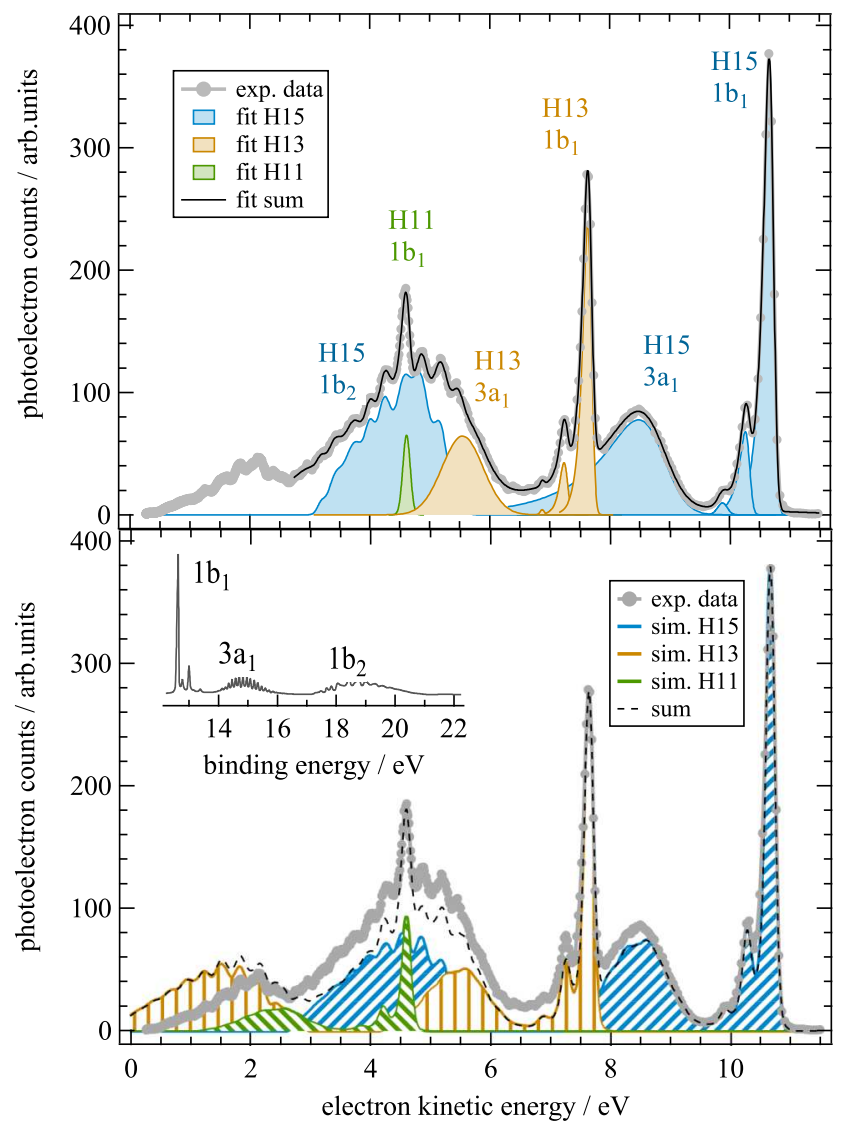

FIG. 9. Photoelectron spectrum (gray circles) of gas-phase water ionized with harmonic orders 11,13 , and 15 . The upper panel shows the fitted total photoelectron signal (black solid line) and its decomposition into the individual photoelectron bands. The lower panel shows the expected photoelectron signal for each harmonic energy. The inset shows the photoelectron spectrum from the literature. ${ }^{40}$ shell photoelectron spectrum of water consists of four bands, associated with the ionization from the $1 b_{1}, 3 a_{1}, 1 b_{2}$, and $2 a_{1}$ orbitals, which are replicated every $3.1 \mathrm{eV}$ by the harmonic orders. The binding energy of the $2 a_{1}$ orbital is too large to be ionized even by the highest harmonic order used here. A high-resolution gas-phase spectrum from the literature, ${ }^{40}$ which is shown in the inset in Fig. 9, was convolved with a Gaussian function ( $0.15 \mathrm{eV}$ FWHM) and used for the simulation of an expected photoelectron spectrum, shown in the lower panel of Fig. 9. The amplitudes of the convolved function were chosen to match the intensity of the $1 b_{1}$ band for each harmonic energy. Thanks to the isolation of the three harmonic orders by the tin filter, the spectral overlap of the individual bands remains manageable for a fitting procedure in the high-kinetic-energy part of the spectrum. However, the vibrationally resolved $1 b_{2}$ band generated by harmonic order 15 poses difficulties to fitting because of overlap with the sharp photoelectron band $1 b_{1}$ generated by harmonic order 11. Nevertheless, all expected photoelectron bands are captured by the fit and all spectral features can be assigned to specific valence orbitals and harmonic orders. The photoelectron spectrum is well represented by the fit and the simulation, which demonstrates the feasibility of using narrow-bandwidth high-harmonic radiation for photoelectron spectroscopy.

From the low kinetic-energy side of the spectrum presented in Fig. 9, it is evident that electrons below 0.5 $\mathrm{eV}$ kinetic energy are still collected. In another publication using the same spectrometer, ${ }^{35}$ we demonstrate that the useful energy range extends to above $60 \mathrm{eV}$ kinetic energy.

\section{Photoelectron spectra of liquid water and solvated sodium iodide}

Photoelectron spectra of microfiltrated liquid water were acquired with a $50 \mu \mathrm{m}$ nozzle, operating at a flow rate of $0.7 \mathrm{ml} / \mathrm{min}$. Sodium chloride at a concentration of $0.05 \mathrm{~mol} / 1$ was added to prevent electro-kinetic charging of the jet. No signal from the sodium cation, or from the chloride anion, was observed under our measurement conditions. The measured photoelectron spectrum generated with harmonic orders $11-15$ is presented in Fig. 10. In contrast to the gas-phase measurements, the XUV photon flux of typically $10^{5}$ photons/shot/harmonic was substantially reduced for the liquid-phase measurements by decreasing the pressure in the gas cell and using an iris aperture to reach an electron count rate of 50-300 electrons per laser shot.

Sharp gas-phase photoelectron signals from the water vapor around the liquid jet are clearly seen in Fig. 10 between the broad photoelectron features of the liquid phase. Due to the very broad liquid-phase water peaks, the photoelectron spectrum of liquid water is less structured than the gas-phase spectrum and peak overlaps pose a challenge to the data analysis. Additionally, secondary electrons produce a broad background at low kinetic energies, which is evident in Fig. 10 as hatched gray curve.

An elaborate fitting procedure would be necessary in order to disentangle the individual spectral components in the liquid water spectra, which in turn would benefit from 


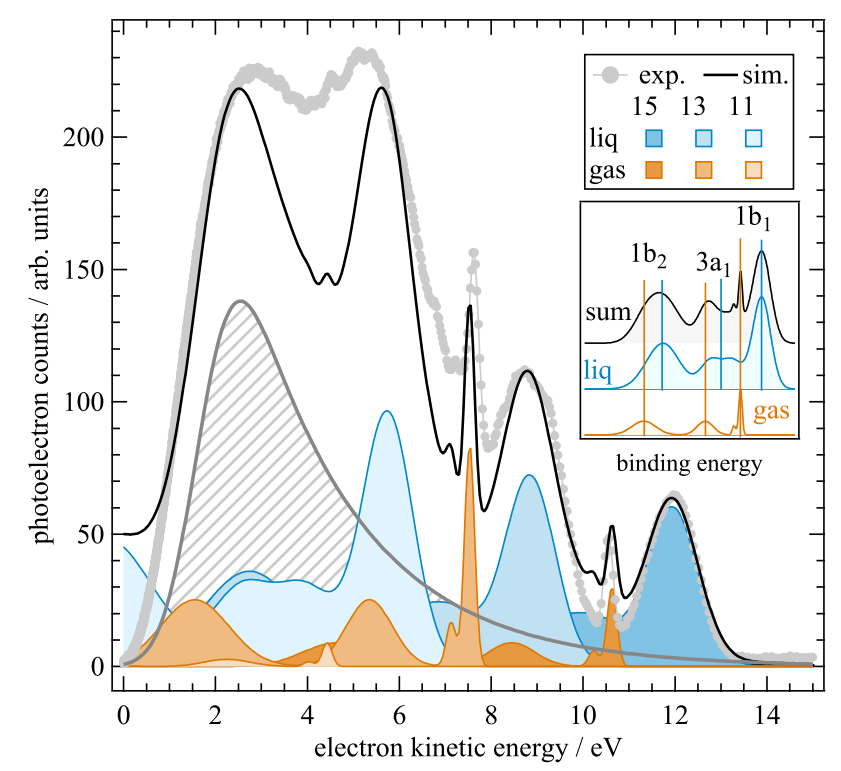

FIG. 10. Photoelectron spectrum of liquid water generated with harmonic orders 11-15 and simulated spectral components. Photoelectron bands from water are shown in blue; bands from the gas phase are shown in orange. The distribution of secondary electrons is represented by the hatched gray curve.

the knowledge of the energy dependence of the ionization cross sections and the high-harmonic energies, intensities, and spectral widths. All these parameters allow to put boundary conditions on the fit which should result in a more robust fit performance. Here, for proof of principle, we used binding energies and peak widths reported in the literature ${ }^{41}$ to simulate a photoelectron spectrum with Gaussian line shapes (Fig. 10). Peak amplitudes have been chosen such that they match the measured intensities well for the $1 b_{1}$ bands and the remaining amplitudes were chosen to match the experimental spectrum. Except for the kinetic energies of the gas-phase signals generated with harmonic orders 11 and 13 , the general shape of the measured photoelectron spectrum can be represented reasonably well with the simulation. Sharp features, namely, the gas- and liquid-phase $1 b_{1}$ orbitals, can be identified and assigned to harmonic orders. Since the liquid jet exhibits a surface potential, the electrons from the gas phase around the grounded jet will travel different trajectories than the electrons of the same kinetic energy from the liquid. The calibration was performed with both the liquid and the gas-phase signals. The background consisting of secondary electrons was represented by an exponentially modified Gaussian. ${ }^{11}$

In the presence of the assisting infrared laser field, sidebands appear in the photoelectron spectrum of liquid water, through the same mechanism as described in Section IV A for the gas phase. The disentanglement of sidebands and principal peaks (from ionization with high-harmonic radiation only) is best accomplished by single-shot acquisition with a chopper blocking every second laser shot in the infrared beam path. The photoelectron spectrum generated without the assisting infrared field is subtracted from the spectrum generated in the presence of the infrared field. The resulting difference spectrum has positive and negative contributions, arising from spectral components dominated by the sideband generation and principal peak depletion, respectively.

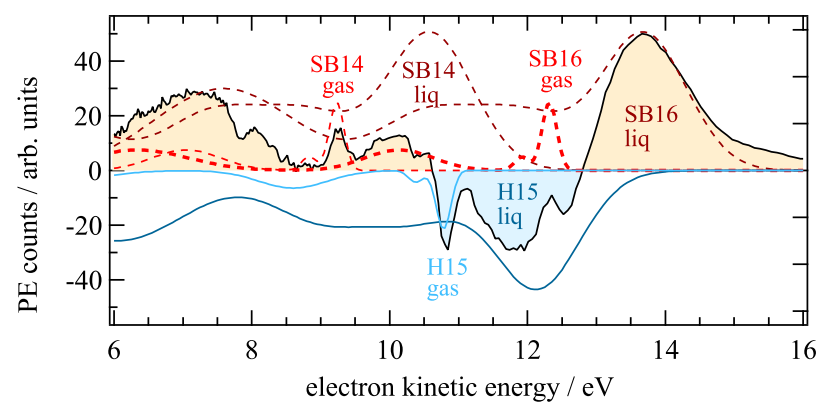

FIG. 11. Difference plot between liquid water ionized with XUV (harmonic orders 11-15) in the presence of an assisting infrared field and liquid water ionized with XUV radiation alone. The filled curve represents the data points; the dashed red curves (positive sign) are the simulated sidebands from the liquid phase. The simulation of liquid- and gas-phase contributions from high-harmonic radiation only is shown in blue solid lines (negative sign).

In Fig. 11, SB16 is associated with the absorption of harmonic order 15 and an additional infrared photon, whereas SB14 is generated through the absorption of harmonic order 15 and the emission of one infrared photon. The latter ionization path interferes with the absorption of harmonic order 13 and an infrared photon and gives rise to an oscillation of SB 14 as a function of the delay between XUV and IR pulses. Both sidebands are clearly visible as positive contributions to the difference spectrum. The principal peak $\mathrm{H} 15$ resulting from ionization of the liquid water with harmonic order 15 is depleted due to population transfer to the sidebands and therefore appears with a negative sign.

With the tin filter, only photon energies up to $23.3 \mathrm{eV}$ are available, and therefore the present approach is best suited for studying outer-valence ionization dynamics. Other metal filters like titanium or zirconium, which transmit at higher photon energies, could be used to access innervalence or core shells. However, valence orbitals with binding energies lower than the HOMO of water can be probed nearly background-free because they appear on the highkinetic-energy edge of the spectrum. We illustrate this case by studying photoemission from a $1 \mathrm{M}$ aqueous sodium iodide solution. The two spin-orbit components of the $5 \mathrm{p}$ shell of iodide have binding energies of $8.03 \mathrm{eV}$ and $8.96 \mathrm{eV}$, respectively. ${ }^{41}$ The measured photoelectron spectrum is shown in Figure 12. Using the tin filter, the highest kinetic energies of the electrons originating from iodide are around $15 \mathrm{eV}$, where the spectrometer resolution is sufficient to resolve the components. The energy difference between the spin-orbit

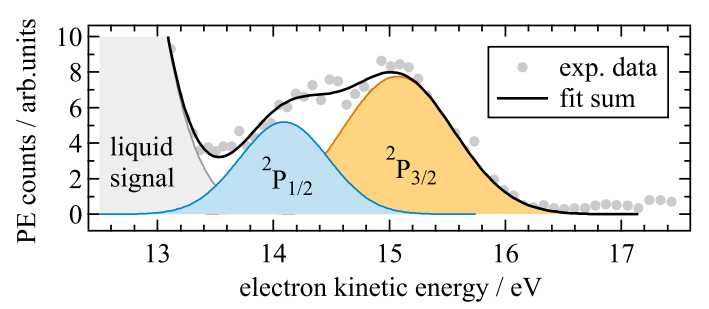

FIG. 12. Photoelectron spectrum of solvated sodium iodide (1 M) ionized with harmonic order 15 . The orange filled curve corresponds to the Gaussian fit to the ${ }^{2} \mathrm{P}_{3 / 2}$ component and the blue filled curve to the ${ }^{2} \mathrm{P}_{1 / 2}$ component. 
components of the iodide anion in our spectra is $(1.0 \pm 0.1) \mathrm{eV}$, which is in good agreement with the value of $(0.93 \pm 0.09) \mathrm{eV}$ reported in the literature. ${ }^{41}$ Standard deviations refer to the fitting of Gaussian line shapes to the experimental data.

\section{CONCLUSIONS}

We have described the design and operation of an attosecond time-resolved experiment for gaseous and liquid samples. The energy resolution of the photoelectron spectrometer was analyzed and it was demonstrated that the spectrometer operates at nearly constant $\Delta E / E$-values in the energy region between $5 \mathrm{eV}$ and $25 \mathrm{eV}$. We have presented the functionality of the setup for recording RABBIT traces, which allowed us to characterize the spectral phase and amplitude of the attosecond pulse train generated in our gas cell. Photoelectron spectra of both liquid and gas-phase water generated with harmonic orders 11,13 , and 15 have been deconvoluted and assigned. Sidebands characteristic of the RABBIT mechanism have been observed. The spin-orbit states associated with the photoionization of the $5 p$ shell of the solvated iodide anion have been identified and analyzed on the high-kinetic-energy side of the photoelectron spectra. This article demonstrates the applicability of our attosecond time-resolved photoelectron spectroscopy setup for both gases and liquids. The present work thus lays the foundations for taking attosecond science into the realm of liquids.

\section{ACKNOWLEDGMENTS}

The authors greatly appreciate discussions with A. Lübcke and the support of D. Stapfer, A. Laso, and A. Schneider. This work was supported by the ETH-FAST initiative as part of the NCCR-MUST programme and an ERC Starting Grant (Project No. 307270-ATTOSCOPE).

${ }^{1}$ S. Neppl, R. Ernstorfer, A. L. Cavalieri, C. Lemell, G. Wachter, E. Magerl, E. M. Bothschafter, M. Jobst, M. Hofstetter, U. Kleineberg, J. V. Barth, D. Menzel, J. Burgdörfer, P. Feulner, F. Krausz, and R. Kienberger, "Direct observation of electron propagation and dielectric screening on the atomic length scale," Nature 517, 342-346 (2015).

${ }^{2}$ A. S. Alnaser, M. Kübel, R. Siemering, B. Bergues, N. G. Kling, K. J. Betsch, Y. Deng, J. Schmidt, Z. A. Alahmed, A. M. Azzeer, J. Ullrich, I. Ben-Itzhak, R. Moshammer, U. Kleineberg, F. Krausz, R. de Vivie-Riedle, and M. F. Kling, "Subfemtosecond steering of hydrocarbon deprotonation through superposition of vibrational modes," Nat. Commun. 5, 3800 (2014).

${ }^{3}$ P. M. Kraus, B. Mignolet, D. Baykusheva, A. Rupenyan, L. Horný, E. F. Penka, G. Grassi, O. I. Tolstikhin, J. Schneider, F. Jensen, L. B. Madsen, A. D. Bandrauk, F. Remacle, and H. J. Wörner, "Measurement and laser control of attosecond charge migration in ionized iodoacetylene," Science 350, 790-795 (2015).

${ }^{4}$ M. Schultze, K. Ramasesha, C. D. Pemmaraju, S. A. Sato, D. Whitmore, A. Gandman, J. S. Prell, L. J. Borja, D. Prendergast, K. Yabana, D. M. Neumark, and S. R. Leone, "Ultrafast dynamics. Attosecond band-gap dynamics in silicon," Science 346, 1348-1352 (2014).

${ }^{5}$ M. H. Elkins, H. L. Williams, A. T. Shreve, and D. M. Neumark, "Relaxation mechanism of the hydrated electron," Science 342, 1496-1499 (2013).

${ }^{6}$ H. J. Wörner, J. B. Bertrand, B. Fabre, J. Higuet, H. Ruf, A. Dubrouil, S. Patchkovskii, M. Spanner, Y. Mairesse, V. Blanchet, E. Mével, E. Constant, P. B. Corkum, and D. M. Villeneuve, "Conical intersection dynamics in $\mathrm{NO}_{2}$ probed by homodyne high-harmonic spectroscopy," Science 334, 208 (2011).

${ }^{7}$ J. Mauritsson, T. Remetter, M. Swoboda, K. Klünder, A. L'Huillier, K. J. Schafer, O. Ghafur, F. Kelkensberg, W. Siu, P. Johnsson, M. J. J. Vrakking,
I. Znakovskaya, T. Uphues, S. Zherebtsov, M. F. Kling, F. Lépine, E. Benedetti, F. Ferrari, G. Sansone, and M. Nisoli, "Attosecond electron spectroscopy using a novel interferometric pump-probe technique," Phys. Rev. Lett. 105, 053001 (2010).

${ }^{8}$ S. Baker, J. S. Robinson, C. A. Haworth, H. Teng, R. A. Smith, C. C. Chirila, M. Lein, J. W. G. Tisch, and J. P. Marangos, "Probing proton dynamics in molecules on an attosecond time scale," Science 312, 424-427 (2006).

${ }^{9}$ M. Hentschel, R. Kienberger, C. Spielmann, G. A. Reider, N. Milosevic, T. Brabec, P. Corkum, U. Heinzmann, M. Drescher, and F. Krausz, "Attosecond metrology," Nature 414, 509-513 (2001).

${ }^{10} \mathrm{~T}$. Brabec and F. Krausz, "Intense few-cycle laser fields: Frontiers of nonlinear optics," Rev. Mod. Phys. 72, 545-591 (2000).

${ }^{11}$ M. Faubel, B. Steiner, and J. P. Toennies, "Photoelectron spectroscopy of liquid water, some alcohols, and pure nonane in free micro jets," J. Chem. Phys. 106, 9013-9031 (1997)

${ }^{12}$ B. Winter and M. Faubel, "Photoemission from liquid aqueous solutions," Chem. Rev. 106, 1176-1211 (2006)

${ }^{13}$ O. Link, V.-M. Esteban, E. Lugovoj, Y. Liu, K. Siefermann, M. Faubel, H. Grubmüller, R. B. Gerber, Y. Miller, and B. Abel, "Ultrafast phase transitions in metastable water near liquid interfaces," Faraday Discuss. 141, 67 (2009).

${ }^{14}$ F. Buchner, A. Nakayama, S. Yamazaki, H.-H. Ritze, and A. Lübcke, "Excited-state relaxation of hydrated thymine and thymidine measured by liquid-jet photoelectron spectroscopy: Experiment and simulation," J. Am. Chem. Soc. 137, 2931-2938 (2015).

${ }^{15}$ A. Beloqui Redondo, I. Jordan, I. Ziazadeh, A. Kleibert, J. B. Giorgi, H. J. Wörner, S. May, Z. Abbas, and M. A. Brown, "Nanoparticle-induced charge redistribution of the air-water interface," J. Phys. Chem. C 119, 2661-2668 (2015).

${ }^{16} \mathrm{R}$. Seidel, S. Thürmer, J. Moens, P. Geerlings, J. Blumberger, and B. Winter, "Valence photoemission spectra of aqueous $\mathrm{Fe}^{(2+/ 3+)}$ and $\left[\mathrm{Fe}(\mathrm{CN})_{6}\right]^{(4-/ 3-)}$ and their interpretation by DFT calculations," J. Phys. Chem. B 115, 11671-11677 (2011).

${ }^{17}$ C. Bressler, C. Milne, V.-T. Pham, A. Elnahhas, R. M. van der Veen, W. Gawelda, S. Johnson, P. Beaud, D. Grolimund, M. Kaiser, C. N. Borca, G. Ingold, R. Abela, and M. Chergui, "Femtosecond XANES study of the light-induced spin crossover dynamics in an iron(II) complex," Science 323, 489-492 (2009).

${ }^{18}$ E. F. Aziz, N. Ottosson, S. Bonhommeau, N. Bergmann, W. Eberhardt, and M. Chergui, "Probing the electronic structure of the hemoglobin active center in physiological solutions," Phys. Rev. Lett. 102, 068103 ( 2009).

${ }^{19}$ E. Suljoti, R. Garcia-Diez, S. I. Bokarev, K. M. Lange, R. Schoch, B. Dierker, M. Dantz, K. Yamamoto, N. Engel, K. Atak, O. Kühn, M. Bauer, J.-E. Rubensson, and E. F. Aziz, "Direct observation of molecular orbital mixing in a solvated organometallic complex," Angew. Chem., Int. Ed. 52, 9841-9844 (2013)

${ }^{20}$ T. Horio, H. Shen, S. Adachi, and T. Suzuki, "Photoelectron spectra of solvated electrons in bulk water, methanol, and ethanol," Chem. Phys. Lett. 535, 12-16 (2012).

${ }^{21}$ M. A. Brown, A. B. Redondo, I. Jordan, N. Duyckaerts, M.-T. Lee, M. Ammann, F. Nolting, A. Kleibert, T. Huthwelker, J.-P. Mächler, M. Birrer, J. Honegger, R. Wetter, H. J. Wörner, and J. A. van Bokhoven, "A new endstation at the swiss light source for ultraviolet photoelectron spectroscopy, X-ray photoelectron spectroscopy, and X-ray absorption spectroscopy measurements of liquid solutions," Rev. Sci. Instrum. 84, 073904 ( 2013).

${ }^{22}$ B. Winter, "Liquid microjet for photoelectron spectroscopy," Nucl. Instrum. Methods Phys. Res., Sect. A 601, 139-150 (2009).

${ }^{23}$ A. Kothe, J. Metje, M. Wilke, A. Moguilevski, N. Engel, R. Al-Obaidi, C. Richter, R. Golnak, I. Y. Kiyan, and E. F. Aziz, "Time-of-flight electron spectrometer for a broad range of kinetic energies," Rev. Sci. Instrum. 84, 023106 (2013).

${ }^{24}$ F. Buchner, A. Lübcke, N. Heine, and T. Schultz, "Time-resolved photoelectron spectroscopy of liquids," Rev. Sci. Instrum. 81, 113107 (2010).

${ }^{25}$ Y.-I. Suzuki, H. Shen, Y. Tang, N. Kurahashi, K. Sekiguchi, T. Mizuno, and T. Suzuki, "Isotope effect on ultrafast charge-transfer-to-solvent reaction from $\mathrm{I}^{-}$to water in aqueous $\mathrm{NaI}$ solution," Chem. Sci. 2, 1094 (2011).

${ }^{26}$ K. R. Siefermann, Y. Liu, E. Lugovoy, O. Link, M. Faubel, U. Buck, B. Winter, and B. Abel, "Binding energies, lifetimes and implications of bulk and interface solvated electrons in water," Nat. Chem. 2, 274-279 (2010).

${ }^{27}$ C. A. Arrell, J. Ojeda, M. Sabbar, W. A. Okell, T. Witting, T. Siegel, Z. Diveki, S. Hutchinson, L. Gallmann, U. Keller, F. van Mourik, R. T. Chapman, C. Cacho, N. Rodrigues, I. C. E. Turcu, J. W. G. Tisch, E. 
Springate, J. P. Marangos, and M. Chergui, "A simple electron time-of-flight spectrometer for ultrafast vacuum ultraviolet photoelectron spectroscopy of liquid solutions," Rev. Sci. Instrum. 85, 103117 (2014).

${ }^{28}$ Y. Tang, H. Shen, K. Sekiguchi, N. Kurahashi, T. Mizuno, Y.-i. Suzuki, and T. Suzuki, "Direct measurement of vertical binding energy of a hydrated electron," Phys. Chem. Chem. Phys. 12, 3653-3655 (2010).

${ }^{29}$ F. Buchner, H.-H. Ritze, J. Lahl, and A. Lübcke, "Time-resolved photoelectron spectroscopy of adenine and adenosine in aqueous solution," Phys. Chem. Chem. Phys. 15, 11402-11408 (2013).

${ }^{30}$ Y. Tang, Y. Suzuki, H. Shen, K. Sekiguchi, N. Kurahashi, K. Nishizawa, P. Zuo, and T. Suzuki, "Time-resolved photoelectron spectroscopy of bulk liquids at ultra-low kinetic energy," Chem. Phys. Lett. 494, 111-116 (2010).

${ }^{31}$ P. M. Paul, E. S. Toma, P. Breger, G. Mullot, F. Auge, P. Balcou, H. G. Muller, and P. Agostini, "Observation of a train of attosecond pulses from high harmonic generation," Science 292, 1689-1692 (2001).

${ }^{32} \mathrm{P}$. Kruit and F. H. Read, "Magnetic field paralleliser for $2 \pi$ electronspectrometer and electron-image magnifier," J. Phys. E: Sci. Instrum. 16, 313-324 (1983).

${ }^{33}$ M. Fiess, M. Schultze, E. Goulielmakis, B. Dennhardt, J. Gagnon, M. Hofstetter, R. Kienberger, and F. Krausz, "Versatile apparatus for attosecond metrology and spectroscopy," Rev. Sci. Instrum. 81, 093103 (2010).

${ }^{34}$ R. Locher, M. Lucchini, J. Herrmann, M. Sabbar, M. Weger, A. Ludwig, L. Castiglioni, M. Greif, M. Hengsberger, L. Gallmann, and U. Keller, "Versatile attosecond beamline in a two-foci configuration for simultaneous time-resolved measurements," Rev. Sci. Instrum. 85, 013113 (2014).
${ }^{35}$ M. Huppert, I. Jordan, and H. J. Wörner, "Attosecond beam-line with actively-stabilized and spatially separated beam paths," Rev. Sci. Instrum. 86, 123106 (2015).

${ }^{36}$ E. B. Saloman, "Energy levels and observed spectral lines of xenon, Xe I through XeLIV," J. Phys. Chem. Ref. Data 33, 765-921 (2004)

${ }^{37}$ K. Klünder, J. M. Dahlström, M. Gisselbrecht, T. Fordell, M. Swoboda, D. Guénot, P. Johnsson, J. Caillat, J. Mauritsson, A. Maquet, R. Taïeb, and A. L'Huillier, "Probing single-photon ionization on the attosecond time scale," Phys. Rev. Lett. 106, 143002 (2011).

${ }^{38}$ J. M. Dahlström, A. L'Huillier, and A. Maquet, "Introduction to attosecond delays in photoionization," J. Phys. B 45, 183001 (2012).

${ }^{39}$ J. Mauritsson, M. B. Gaarde, and K. J. Schafer, "Accessing properties of electron wave packets generated by attosecond pulse trains through timedependent calculations," Phys. Rev. A 72, 013401 (2005).

${ }^{40} \mathrm{~K}$. Kimura, Handbook of HeI Photoelectron Spectra of Fundamental Organic Molecules: Ionization Energies, Ab Initio Assignments, and Valence Electronic Structure for 200 Molecules (Japan Scientific Societies Press, 1981)

${ }^{41}$ N. Kurahashi, S. Karashima, Y. Tang, T. Horio, B. Abulimiti, Y.-I. Suzuki, Y. Ogi, M. Oura, and T. Suzuki, "Photoelectron spectroscopy of aqueous solutions: Streaming potentials of $\mathrm{NaX}(\mathrm{X}=\mathrm{Cl}, \mathrm{Br}$, and I) solutions and electron binding energies of liquid water and $\mathrm{X}^{-}$, ” J. Chem. Phys. 140, 174506 (2014).

${ }^{42}$ M. Faubel, S. Schlemmer, and J. P. Toennies, "A molecular beam study of the evaporation of water from a liquid jet," Z. Phys. D 10, 269 (1988). 